\title{
Transcriptome signature of the lactation process in a fat-tailed sheep identifies with integrative approach of RNA-Seq and Supervised Machine Learning models
}

Mohammad Farhadian ( $\square$ Mohammad.farhadian@tabrizu.ac.ir)

University of Tabriz

Seyed Abbas Rafat

University of Tabriz

Bahman Panahi

ABRII

Esmaeil Ebrahimie

The University of Adelaide

Research article

Keywords: RNA-Seq, Milk, Sheep, Gene network, Gene Ontology

Posted Date: July 18th, 2019

DOI: https://doi.org/10.21203/rs.2.11678/v1

License: (c) (1) This work is licensed under a Creative Commons Attribution 4.0 International License.

Read Full License 


\section{Abstract}

Background The mammary gland is a vital organ in mammalian that undergoes a substantial physiological transformation during the lactation process. Understanding the underlying molecular mechanism of the lactation process in the mammary gland is essential to unravel of the complexity of milk production process. This study was investigated the transcriptome profiles of milk between two time point of lactation (day 20 as before peak time point (BF) and day 70 as after peak(AF)) in Iranian fattailed Ghezel sheep breed. Functional impacts of differentially expressed genes (DEGs) between two time points of the lactation were surveyed with Gene Ontology (GO) and Protein-Protein Interaction (PPI) network analysis. Moreover, to identify the transcriptome signature of the lactation process, supervised machine learning algorithms were integrated. Results Totally, 75 genes were defined as DEGs between BF and $\mathrm{AF}$ of lactation. Gene ontology of DEGs mainly enriched in metabolic process and oxidative phosphorylation. Moreover, PPI networks analysis highlighted the contribution of peroxisome proliferatoractivated receptors (PPAR) signaling in the lactation process, oxidative phosphorylation and metabolic pathways. These pathways mainly involved in the quality of dairy products and energy generation for milk production. Dissection of DEGs also indicated that the genes encoding for caseins, whey proteins, and enzymes lactose synthesis are dominantly up-regulated after the lactation peak. More interestingly, the genes involved in fat metabolism dominantly down-regulated after the lactation peak. Contribution of nineteen DEGs such as HNRNPA2, CHCHD2, COX1, COX2, GTF3C3, and MRPL11 has verified with more than seven attribute ranking algorithms, which are being proposed as a signature of the lactation process in Ghezel sheep. Conclusions The results of this study highlighted the energy generation, development of immunity, and activation of the immune system in lactation process. To best of our knowledge, this is the first study comparing the underlying mechanism of Ghezel sheep before and after the lactation peak and provides new insight regarding the transcriptional responses for the lactation process.

\section{Background}

Milk is a white liquid food and nutrient-rich that is consumed by mammalian infants and secret by mammalian during the lactation period. The lactation stage can be divided into three phases; the early, mid and late lactation [1]. Human and cow milk [2] and sheep milk [3] have a significant difference in the physicochemical features. Also, there are many differences between each stage of lactation processes in different phases. Fat and protein contents of sheep milk are higher than goat and cow milk. Moreover, in comparison with cows, buffaloes, and goats milks, lactose content of sheep milk is higher [4].

Milk contains a different percentage of fat, protein, lactose, casein and whey protein in early, mid and late lactation in sheep [3], cow and human [5]. These sheep milk properties make it appropriate for dairy products such as yoghurt and cheese making.

The milk transcriptome has been characterized in human [6, 7], bovine [8, 9] and sheep [10]. In Spanish sheep breed the number of 573 DEGs introduced across lactation points (10, 50, 120 and 150 days), with 
the most considerable divergences being found, between day 10 and day 150 [10]. It has been also reported that immune and stress responsive genes are important pathways in the lactation process [10].

Transcriptome profiling technologies, such as cDNA library, microarray, and RNA-sequencing is versatile tools to get new insight of underlying mechanism of different biological functions and cellular activities [11-16]. To tarnscriptome level study of lactation process, mammary gland biopsies [6], milk fat globules (MFGs) [7] and milk somatic cells (MSCs) [8] is commonly used.

Among them, MFG transcriptome represents the RNA profiles from mammary epithelial cells (MEC) and mammary gland in lactation processes studies $[7,9,17,18]$.

Sheep milk is mainly used for cheese production, so its quality directly influences cheese yield [19]. Ghezel sheep has originated from northwestern provinces of Iran (East and west Azerbaijan) and west of Turkey and is commonly tolerated to dry and cold circumstances [20]. Ghezel sheep's is commonly used for its dairy products characteristics, Lighvan cheese is a famous traditional cheese made from Ghezel sheep milk in the village of Lighvan located in the northwest Iran, East Azerbaijan province [21]. So, it is an ideal model breed for studding the milk production underlying mechanisms.

In this study, RNA-Seq transcriptome profiling were integrated with Machine Learning modeling to obtain the signature of two distinct time point of lactation (before peak and after peak lactation) in Ghezel sheep.

\section{Methods}

Animals and sampling

Two healthy Ghezel sheep at the second lactation stage were involved in this study. These sheep were kept in free stall housing; feeding with the same rations and without water restrictions. Milk samples were collected with a hand at two lactation stages: the before peak stage (on day 20 after lambing) and after peak stage (on day 75 after lambing). Collected samples were stored in sterile and RNase-free 50-ml tubes for MFG extraction.

\section{Collection of MGFs and RNA isolation}

Samples were centrifuged at $2000 \mathrm{~g}$ for $10 \mathrm{~min}$ at four $0^{\mathrm{C}}$ in order to isolate milk fat. The supernatant fat

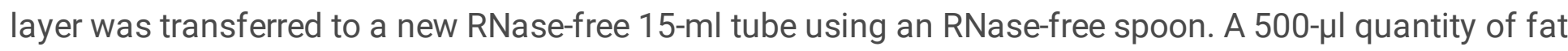
was put into a $15-\mathrm{ml}$ tube, and $1.5 \mathrm{ml}$ of Trizol reagent (Invitrogen Life Technology) was added to dissolve the fat layer. Total RNA from MFG was extracted by using a TRIzol LS solution (Invitrogen Life Technologies Inc.) following the manufacturer's protocol, with minor modifications. Briefly, to remove lipids, centrifugation was performed at $12,000 \times \mathrm{g}$ for $10 \mathrm{~min}$ at $4^{\circ} \mathrm{C}$. A $400-\mu \mathrm{L}$ quantity of chloroform was then added to the remaining solution and the mix was shaken vigorously. After centrifugation, the upper aqueous layer (approximately 60\%), which were contained RNA, was carefully pipetted, and RNA was 
precipitated by adding $750 \mu \mathrm{L}$ of isopropanol/1 mL of TRIzol LS used for extraction. After centrifugation $\left(12,000 \times \mathrm{g}\right.$ for $10 \mathrm{~min}$ at $\left.4^{\circ} \mathrm{C}\right)$, RNA pellets were washed with $75 \%$ ethanol, dissolved in $30 \mu \mathrm{L}$ of RNasefree water, and finally stored at $-80^{\circ} \mathrm{C}$ until further processing. Genomic DNA contaminations were removed by DNase I (Qiagen, Hilden, Germany). Then, the quality and quantity of isolated RNA were surveyed with agarose gel electrophoresis and Nano drop spectrophotometry, respectively.

RNA sequencing and Data analysis

The RNA integrity value (RIN) of the samples selected to be sequenced ranged between 7 and 8 . Pairedend libraries fragmented into short fragments (200-700 bp) by using the fragmentation buffer. The fragments were sequenced on an Illumina Hi-Seq 4000 sequencer, according to the manufacturer's instructions (BGI). For each library, 60 million paired-end 100 bp reads were generated during the sequencing run. The quality of raw data was evaluated using FastQC (Version 0.11.8) (ANDREWS et al. 2016) program. Reads with quality score below 30 were trimmed out using PRINSEQ [22] and Trimmomatics [23]. Then, clean reads were mapped to the sheep reference genome Oar_v3.1 (available at Ensemble) using TOPHAT (v2.1.1) (KIM et al. 2013). Transcripts were assembled by Cufflinks (version 2.2.0) [24], then Cufmerge (version 2.1.1) [24] was used for merging into a single transcriptome annotation. Finally, cuffdiff (version 2.2.1) was used to detect DEGs between the BF and AF samples. Differentially expressed genes at each lactation time point using cummeRbund ( $R$ package), gene length and library size were normalized using (Fragments Per Kilobase Million) FPKM index [10].

Gene Ontology enrichment of DEGs

To analyze the functions of DEGs, GO enrichment of DEGs was performed in three Biological processes, molecular function and cellular component categories [25]. The adj-value $\leq 0.05$ was set as cutoff threshold for enrichment analysis.

Protein-Protein Network analysis

To better understanding of the dynamic process of a complex process such as lactation, information about the functions of genes/proteins is needed[15]. In this regard, the protein-protein interaction network of DEGs was firstly constructed using the STRING (https://string-db.org/) database. Experimentally validated, text mining, databases, co-expression, and neighborhood interactions were considered for the network construction [25]. Then, to define the functional modules, constructed networks were clustered with the $\mathrm{K}$ means algorithm. $\mathrm{K}$-means is a popular clustering algorithm which is widely used in anomalybased intrusion detection. It tries to classify a given data set into $\mathrm{k}$ (a predefined number) categories [26].

Selection of transcriptome signature using the Attribute weighting algorithms

To identify the transcriptome signature of the lactation process, attribute weighting algorithms were implied on genes with significant expression in two time point of lactation. First, the two-step standardization procedure was carried out on expression data as previously proposed [27-30]. Then, stage of lactation (AF and BF) and FPKM values of DEGs was set as label attribute target, respectively. 
Ten attribute weighting algorithms including the Information Gain, Information Gain Ratio, Chi-Squared, Deviation, Rule, SVM, Gini Index, Uncertainty, Relief, and PCA were separately applied on the DEGs data set. The DEGs with weighting value higher than 0.7 were considered as signature [31-33].

\section{Results}

Summary statistics for the RNA-Seq data

Milk samples of Ghezel sheep from two lactation time points including the D20 (BF period) and D75 of lactation (AF period) with two biological replicates were sequenced. A total of $243,063,312$ paired-end reads, $\sim 60$ million reads per sample were obtained from transcriptome sequencing of milk samples $(n=4)$. Alignment of the reads to the Ovis arise genome Oar_v4.1 was $73.25 \%$ at mean (Table 1$)$.

Table 1: General statistic of reads in pre and post-trimming and mapping results

AP: After Peak, BP: Before Peak

The mapping ratio for each samples were described in Table 2. According to the FPKM value, mapped genes were divided into the high, moderate and low expression for genes with $500>\mathrm{FPKM}, 10<\mathrm{FPKM}$ $<500$ and $<10$ FPKM, respectively. Genes with $>0.02$ FPKM were considered as a significant expression [8]. Totally, 23148 and 22362 genes were defined as expressed genes in BF and AF, respectively.

Table 2: RNA-Seq gene expression distribution for the Ghezel sheep breed in the BF and AF of lactation

It is apparent from Table 2 that the majority of the genes have had low expression across lactation stages.

\section{Analysis of the some most expressed genes in each stage of lactation}

The normalization index makes simplify the comparison of expressed genes within a sample [34], so FPKM index were used to determine the DEGs. Table 2 shows the number of genes with the highest FPKM $(\geq 500)$ at BF and AF of lactation. Distribution of FPKM values were provided in Figure 1. It is apparent from figure 1 that ATP6, PAEP, CSN1S1, CSN2, LALBA, RPS29, CYTB and CSN1S2 genes have increased expression in progress of lactation. Whereas, decreased expression of ND1, ND6 and ZNF33B genes were shown in the $\mathrm{AF}$ in comparison with $\mathrm{BF}$ of lactation. 
Differential expression analysis with edgeR package has defined the 75 genes as DEGs between two stages of lactation. Among these DEGs, 60 and 15 genes were up- and down-regulated, respectively (Fig. 2)

\section{Gene ontology}

To indicate possible functions of DEGs identified in the milk samples, the genes were classified into three main categories (biological process, molecular function, cellular component) according to the GO algorithm (Fig 3). In the biological process category, "oxidation-reduction process", "metabolic process", "cation transport", and "oxidative phosphorylation" dominantly enriched.

In the "Cellular components" category "cell", "membrane part" "cytoplasmic part", and "mitochondrial membrane" were dominant. Finally, in the "molecular function" category "catalytic activity", "oxidoreductase activity", and "binding" were enriched.

\section{Expression of genes encoding for caseins, whey proteins and lactose synthesis pathway enzymes}

Caseins (CN) and whey proteins are two common milk proteins. Caseins such as as $1-\mathrm{CN}$, as $1-\mathrm{CN}, \beta-\mathrm{CN}$, and $₫-C N$ are encoded by CSN1S1, CSN1S2, CSN2, and CSN3, respectively [35]. Most of the genes involved in casein synthesis were unregulated in the BF time point of lactation. However, the CSN2 and CSN3 genes have down-regulated in BF time point of lactation.

Lactose is encoded by B4GALTL and LALBA. As shown in Figure 4, both B4GALTL and LALBA genes that are involved in lactose synthesis had increased expression in BF versus AF period of lactation. The expression of lactose encoded genes patterns showed a positive correlation with the lactose concentration in milk, which is decreased with advanced lactation $[8,36]$.

\section{The expression profile of the genes involved in fat metabolism}

Most of the key genes involved in milk fat metabolism are differentially expressed in FMG during lactation stages (Fig 5). Fatty acid uptake is the first process in milk fat metabolism. Lipoprotein lipase (LPL) and very low-density lipoprotein receptor (VLDLR) binds and increases the catabolism of apolipoprotein Etriglyceride- rich lipoproteins [8]. LPL indicated high expression in BF period and along with lactation progress, its expression decreased. VLDLR also showed a similar expression pattern.

De novo fatty acid synthesis is the second process of fat metabolism. The rate-limiting enzyme in fatty acid synthesis is encoded by Acetyl-coenzyme A carboxylase alpha (ACACA). Results showed that ACACA expression is decreased after the lactation peak. Fatty acid synthase (FASN) gene is other important gene 
in de novo fatty acid synthesis, which showed similar expression pattern with ACACA during two time points of lactation peak.

Coordinate expression of ACACA and FASN genes in lactation stages has been also reported by [37]. Next process in milk fat metabolism is fatty acid desaturation. Generally, fatty acids can be divided into two groups: mono fatty acids and very long fatty acids. The mono fatty acids unsaturated by StearoylCoA desaturase (SCD1) [38, 39]; whereas, desaturation of very long fatty acids is performed by fatty acid desaturase 1 and two encoded by FADS1 and FADS2. All of the mentioned genes involved in fatty acid desaturation showed increased expression in $\mathrm{BF}$ and decreased expression in the AF period. This results in agreement with the results of previous findings in bovine [8, 40] and rat [41].

In the fatty acid esterification step, the number of four genes include Glycerol-3-phosphate 0acyltransferase (GPAM), 1- acylglycerol-3-phosphate 0-acyltransferase 6 (AGPAT6) and Diacylglycerol transferase 1 and 2 (DGAT1, DGAT2) genes. GPAM and AGPAT6 genes encode for the esterification in mono- and diacylglycerol synthesis whereas DAGT1 and DAGT2 genes encode for triacylglycerol (TAG) synthesis. All of these genes, except DAGT2, have showed increased expression in BF and decreased expression in AF.

One of the most important processes in milk fat metabolism is fatty acid esterification. The key genes involved in this step are Sterol regulatory element-binding proteins 1 and 2 (SREBF1, SREBF2), Insulin-induced 1 and two genes (INSIG1, INSIG2), and sterol-sensing proteins (SCAP). All of the genes involved in fatty acid esterification showed increased and decreased expression in BF and AF time point of lactation peak, respectively. It has been proposed that SREBF2 and SREBF2 play an important role in transcription regulation of fatty acid esterification in mouse [42] [43]. The final process in fat milk metabolism is milk fat secretion where Butyrophilin (BTN1A1) and Xanthine dehydrogenase $(\mathrm{XDH})$ are main proteins involved in [40]. These genes showed higher expression in BF time point and with the progress of lactation, their expression decreased. Most of the genes involved in milk fat metabolism in MFG samples of Ghezel sheep breed had higher expression in BF rather than AF period.

\section{Gene network}

Functional impacts of DEGs were also surveyed in system levels analysis with PPI network construction. The constructed network was consisting of 55 nodes (DEGs) and 182 edges which strength of interaction score set on $>0.4$ (Fig 6). Disconnected nodes in the network were removed. The generated network is clustered into three functional modules. The first module which highlighted with blue color, annotated in oxidative phosphorylation and metabolic pathways. The second module annotated as PPAR signaling pathway based on KEGG database.

Ribosomal pathway includes RPL and RPS gene family which they have an important role in mammary gland development $[44,45]$ and also, reported that the growth of the mammary gland is a critical process in milk production [46]. 
Finally, the third module enriched in ribosome pathway. Peroxisome proliferator-activated receptors (PPARs) are nuclear hormone receptors that are activated by fatty acids and their derivatives [47].

\section{Attribute weighting algorithms and ranking of DEGs}

Ten different Attribute weightings (AWs) model was applied to identify important DEGs. Data were normalized and were used for model construction. All weighted values ranged between 0 and 1.0. The 1.0 value indicates that a given gene is an important gene. FTH1 gene was the most important gene pointed out by $80 \%$ of the AWs (8 from 10 AWs); followed by HNRNPA2, CHCHD2, COX1, COX2, GTF3C3, MRPL11, RPL12, RPL14, RPL18A, RPL22, RPL32, RPL37, RPL8, RPS10, RPS11, RPS21, and SLC25A4. A table containing the DEGs with all weights given by 10 AWs was shown in a supplementary file (Supplementary 1). A complete list of high relevant genes that were confirmed by the majority of AWs (with a weight above 0.7 ) is presented in Table 3.

Table 3: the most important attributes (DEGs introduced by analysis of sheep MFGs) ranked based on 10 AWs

\section{Discussion}

Studying of milk transcriptome profiles in needed to identify the candidate genes in milk production traits. Among the different of RNA milk transcriptome sources, MFGs reported that is an appropriate source than others [9]. The average expression levels for Ghezel sheep were in agreement with previous reports on the milk transcriptome of the other ruminant species $[8,10,48]$. About $65 \%$ of the transcripts were annotated as intergenic transcripts. It is also in line with the results of ovine muscle transcriptome of small-tailed Han and Dorper sheep [49] and milk transcriptome of Churra and Assaf sheep breeds [10]. It seems there is need to do efforts for the completion and improvement of sheep transcriptome annotation.

The FPKM index distribution were applied for identify the DEGs between two time points of lactation.

Results of GO results, Oxidation-reduction (redox status) enriched in BP category, is the most important physicochemical features in quality and flavor determination of dairy products [50]. All of the genes involved in the "redox" process has up regulated in BF which milk fat is higher than the AF time point of lactation. Other term activates is "oxidative phosphorylation" process which contains a series of ATPases in the metabolic pathway to energy generation [51], and milk production is a process that needs high energy $[52,53]$. Less oxidative phosphorylation is a major mechanism contributing to the low milk production in mammalian. Some genes like COX and ND family genes which are involved in the oxidative phosphorylation pathway, have up-regulated in BF time point of lactation. It has been also demonstrated that redox status has a significant positive correlation with fat content in milk [54] which is in agreement with the results of this study. Oxidative phosphorylation process which main process for providing energy 
for milk production take place in mitochondrial [55]. In this study we used MFGs as a RNA source to the evaluation of milk profile gene expression. Milk fat global is the most important part of the milk and some milk fat globules secreted from the cytoplasm [56]. The enriched binding term in milk refers to binding some mineral and enzyme activity in the milk [57,58]. Results of $G O$ analysis confirmed the functional role of the DEGs on milk production. The biological importance of "Redox" and "Oxidative phosphorylation" is in the quality of dairy product and energy generation for milk production.

Among the CN gene family, CSN2 gene showed the highest expression in Ghezel milk. It is in agreement with the caseins profiles which has been reported by [59]. Moreover, expression profiles of casein genes were similar to the somatic cell transcriptome of bovine milk [8]. $\beta$-lactoglobulin and a-lactalbumin are two major components of whey proteins which are encoded by the LGB and LALBA genes, respectively. The LGB gene has not annotated in the sheep genome. Similar expression pattern were shown between LALBA and CN genes (Figure 4). Whey proteins and lactose synthesis involved genes down-regulated after the lactation peak (Figure 3 ). It is, in contrast, with the previous reports $[8,10]$.

Previous studies have been reported that during the lactation progress, expression of whey proteins and lactose synthesis in the Assaf and Churra sheep breed are not changed $[8,10]$. In contrast, results of our study showed that the $\mathrm{CN}$ and whey genes are down-regulated in BF time points. It has been indicated that the total milk protein from early to end lactation has increased rate [60]. It is may be due to cleaving of caseins and whey proteins are broken into bio-active peptides in the early lactation, so their concentration is not reflected in milk proteins profiles [61, 62].

Based on the results of network analysis, "oxidative phosphorylation", is the main function for energy generation for milk production [55].PPAR pathway includes three subtypes ((PPARalpha, beta/delta, and gamma) which encoded by specific genes and bound fatty acids [47]. PPARalpha plays a role in cellular lipids by gene expression involved in lipid metabolism and loss of this expression in mammary epithelial increase immune risks in the mammary gland [63]. Lipid oxidation and cell proliferation is the PPARbeta/delta function [64]. PPARgamma is involved in adipocyte differentiation promotion to enhance blood glucose uptake [65].

attribute weighting algorithms results revealed that the FTH1 gene is one the important and the importance of these genes has been previously highlighted by microarray meta-analysis [46]. Also the importance of theses gene in lactation process of goat milk has been confirmed by RNA-seq method [66]. RPL and RPS gene family which are an important part of the eukaryotic ribosome and may also have an important role in mammary development $[44,45]$, is defined as another signature for lactation process. Applying eight statistically different attribute weighting algorithms and selection of the key features based on the overall (intersection) of these algorithms reinforced the importance of the selected genes.

\section{Conclusion}

To best of our knowledge, this is the first study that transcriptome of Ghezel sheep MFGs in two distinct lactation periods were profiled with RNA-Seq technology. Results of our analysis defined the 75 genes as 
responsive genes between two time points of lactation peak. Among them, caseins, whey proteins, lactose biosynthesis, and fat metabolism involved genes, have significantly up-regulated before lactation peak. Network analysis also highlighted the "oxidative phosphorylation", "metabolic pathways" and "PPAR signaling pathways" contribution in lactation process. Integration of data mining approaches with RNA-Seq technology proposed that the genes involved in energy generation, development and activation of the immune system as transcriptome signature of lactation process in Ghezel sheep MFGs. Our novel integrated approach provides new avenue to unravel of underlying transcriptome mechanism and proposed some transcriptome signature of lactation process for future studies.

\section{Abbreviations}

BF: Before peak; AF: After peak; DEGs: Differentially expressed genes; PPI: Protein-Protein Interaction; PPAR: Peroxisome proliferator-activated receptors; GO: Gene Ontology; MFGs: Milk fat globules; MSCs: Milk somatic cells; MECs: Mammary epithelial cells; RIN: RNA integrity value; FPKM: Fragments per Kilobase Million; CN: Caseins; LPL: Lipoprotein lipase; VLDLR: Very low-density lipoprotein receptor; ACACA: Acetyl-coenzyme A carboxylase alpha; FASN: Fatty acid synthase; SCD1: Stearoyl-CoA desaturase; GPAM: Glycerol-3-phosphate 0-acyltransferase; AGPAT6: Acylglycerol-3-phosphate 0acyltransferase 6; DGAT1: Diacylglycerol transferase 1; DGAT2: Diacylglycerol transferase 2; TAG: Triacylglycerol; SREBF1: Sterol regulatory element-binding proteins 1; SREBF2: Sterol regulatory elementbinding proteins 2; INSIG1: Insulin-induced 1; INSIG2: Insulin-induced 2; SCAP: Sterol-sensing proteins; BTN1A1: Butyrophilin; XDH: Xanthine dehydrogenase; AWs: Attribute weightings; Redox status: Oxidationreduction.

\section{Declarations}

\section{Acknowledgments}

The authors like to acknowledge the Research Center of computational and Department of Animal science of Tabriz University.

\section{Authors' contributions}

All authors read and approved the final manuscript. MF and BP research concept and design, data analysis and interpretation, wrote the article, and final approval of the article. SR wrote the article. EE data analysis and interpretation, critical revision of the article, and final approval of the article.

\section{Funding}

The authors would like to thank the Iran National Science Foundation (INSF) for supporting this research (Grant No. 95814261). The funding agencies had no role in the study design, data collection and analysis, decision to publish, or preparation of the manuscript. 


\section{Availability of data and materials}

The datasets generated and analyzed during the current study are not publicly available due further analysis will be performed but are available from the corresponding author on reasonable request. Please contact author for data requests.

\section{Authors' contributions}

All authors have read and approved the manuscript. MF: research concept and design, data analysis and interpretation, wrote the article, and final approval of the article. SR: wrote the article. EE and BP: data analysis and interpretation, critical revision of the article, and final approval of the article.

\section{Ethics approval and consent to participate}

Two female sheep were obtained from the local farm in Tabriz University station, Tabriz, Iran. We have obtained the written consent to use the animals in our study from the Tabriz University farm section. Animal use protocol of this project was conducted in accordance with the national guidelines and approved by the Local Ethics Committee University in Tabriz, Iran. At the end of the study, the sheep returned to the Tabriz University farm section

\section{Consent for publication}

Not applicable.

\section{Competing interests}

The authors declare that they have no competing interests

\section{References}

1 Strucken EM, Laurenson YC, Brockmann GA: Go with the flow-biology and genetics of the lactation cycle. Frontiers in genetics 2015, 6:118. 

in proteolysis of sheep milk. Journal of dairy science 2009, 92(1):79-86.

. 4 Graeub BE, Chappell MJ, Wittman H, Ledermann S, Kerr RB, Gemmill-Herren B: The state of family farms in the world. World development 2016, 87:1-15.

. 5 Järvinen K-M, Chatchatee P, Bardina L, Beyer K, Sampson HA: IgE and IgG binding epitopes on alactalbumin and $\beta$-lactoglobulin in cow's milk allergy. International archives of allergy and immunology 2001, 126(2):111-118.

. 6 Lemay DG, Ballard OA, Hughes MA, Morrow AL, Horseman ND, Nommsen-Rivers LA: RNA sequencing of the human milk fat layer transcriptome reveals distinct gene expression profiles at three stages of lactation. PloS one 2013, 8(7):e67531.

. 7 Maningat PD, Sen P, Rijnkels M, Sunehag AL, Hadsell DL, Bray M, Haymond MW: Gene expression in the human mammary epithelium during lactation: the milk fat globule transcriptome. Physiological genomics 2009, 37(1):12-22.

. 8 Wickramasinghe S, Rincon G, Islas-Trejo A, Medrano JF: Transcriptional profiling of bovine milk using RNA sequencing. BMC genomics 2012, 13(1):45.

. 9 Yang J, Jiang J, Liu X, Wang H, Guo G, Zhang Q, Jiang L: Differential expression of genes in milk of dairy cattle during lactation. Animal genetics 2016, 47(2):174-180.

.10 Suárez-Vega A, Gutiérrez-Gil B, Klopp C, Robert-Granie C, Tosser-Klopp G, Arranz JJ: Characterization and comparative analysis of the milk transcriptome in two dairy sheep breeds using RNA sequencing. Scientific reports 2015, 5:18399. 
.11 Bansal M, Belcastro V, Ambesi-Impiombato A, Di Bernardo D: How to infer gene networks from expression profiles. Molecular systems biology 2007, 3(1):78.

.12 Moreno-Risueno MA, Busch W, Benfey PN: Omics meet networks-using systems approaches to infer regulatory networks in plants. Current opinion in plant biology 2010, 13(2):126-131.

. 13 Less H, Angelovici R, Tzin V, Galili G: Coordinated gene networks regulating Arabidopsis plant metabolism in response to various stresses and nutritional cues. The Plant Cel/ 2011, 23(4):1264-1271.

.14 Friedel S, Usadel B, Von Wirén N, Sreenivasulu N: Reverse engineering: a key component of systems biology to unravel global abiotic stress cross-talk. Frontiers in plant science 2012, 3:294.

15 Panahi B, Mohammadi SA, Khaksefidi RE, Fallah Mehrabadi J, Ebrahimie E: Genome-wide analysis of alternative splicing events in Hordeum vulgare: Highlighting retention of intron-based splicing and its possible function through network analysis. FEBS letters 2015, 589(23):3564-3575.

. 16 Panahi B, Mohammadi SA, Ruzicka K, Abbasi Holaso H, Zare Mehrjerdi M: Genome-wide identification and co-expression network analysis of nuclear factor-Y in barley revealed potential functions in salt stress. Physiology and Molecular Biology of Plants 2019, 25(2):485-495.

. 17 Cánovas A, Rincón G, Bevilacqua C, Islas-Trejo A, Brenaut P, Hovey RC, Boutinaud M, Morgenthaler C, VanKlompenberg MK, Martin P: Comparison of five different RNA sources to examine the lactating bovine mammary gland transcriptome using RNA-Sequencing. Scientific reports 2014, 4:5297.

. 18 Brenaut P, Bangera R, Bevilacqua C, Rebours E, Cebo C, Martin P: Validation of RNA isolated from milk fat globules to profile mammary epithelial cell expression during lactation and transcriptional response to a bacterial infection. Journal of dairy science 2012, 95(10):6130-6144. 
. 19 Suárez-Vega A, Gutiérrez-Gil B, Arranz J: Transcriptome expression analysis of candidate milk genes affecting cheese-related traits in 2 sheep breeds. Journal of dairy science 2016, 99(8):6381-6390.

. 20 Tavakkolian J: The genetic resources of native farm animals of Iran. Animal Science Research Institute of Iran, Karaj, Iran(in Persian) 1999.

. 21 Valiloo RH, Rafa SA: Ghezel sheep breeding and traditional cheese making in Iran. FAO, Sustainability Pathways 2009.

. 22 Schmieder R, Lim YW, Rohwer F, Edwards R: TagCleaner: Identification and removal of tag sequences from genomic and metagenomic datasets. BMC bioinformatics 2010, 11(1):341.

. 23 Bolger AM, Lohse M, Usadel B: Trimmomatic: a flexible trimmer for Illumina sequence data. Bioinformatics 2014, 30(15):2114-2120.

. 24 Trapnell C, Roberts A, Goff L, Pertea G, Kim D, Kelley DR, Pimentel H, Salzberg SL, Rinn JL, Pachter L: Differential gene and transcript expression analysis of RNA-seq experiments with TopHat and Cufflinks. Nature protocols 2012, 7(3):562.

. 25 Szklarczyk D, Franceschini A, Wyder S, Forslund K, Heller D, Huerta-Cepas J, Simonovic M, Roth A, Santos A, Tsafou KP: STRING v10: protein-protein interaction networks, integrated over the tree of life. Nucleic acids research 2014, 43(D1):D447-D452.

.26 Le V-H, Kim S-R: K-strings algorithm, a new approach based on Kmeans. In: Proceedings of the 2015 Conference on research in adaptive and convergent systems: 2015. ACM: 15-20.

. 27 Ebrahimi M, Lakizadeh A, Agha-Golzadeh P, Ebrahimie E, Ebrahimi M: Prediction of thermostability from amino acid attributes by combination of clustering with attribute weighting: a new vista in engineering enzymes. PloS one 2011, 6(8):e23146. 
. 28 Ebrahimie E, Nurollah Z, Ebrahimi M, Hemmatzadeh F, Ignjatovic J: Unique ability of pandemic influenza to downregulate the genes involved in neuronal disorders. Molecular biology reports 2015, 42(9):1377-1390.

.29 Mohammadi-Dehcheshmeh M, Niazi A, Ebrahimi M, Tahsili M, Nurollah Z, Khaksefid RE, Ebrahimi $M$, Ebrahimie E: Unified transcriptomic signature of arbuscular mycorrhiza colonization in roots of Medicago truncatula by integration of machine learning, promoter analysis, and direct merging metaanalysis. Frontiers in Plant Science 2018, 9.

.30 Ebrahimie E, Ebrahimi F, Ebrahimi M, Tomlinson S, Petrovski KR: A large-scale study of indicators of sub-clinical mastitis in dairy cattle by attribute weighting analysis of milk composition features: highlighting the predictive power of lactose and electrical conductivity. Journal of Dairy Research 2018, 85(2):193-200.

. 31 Sharifi S, Pakdel A, Ebrahimi M, Reecy JM, Farsani SF, Ebrahimie E: Integration of machine learning and meta-analysis identifies the transcriptomic bio-signature of mastitis disease in cattle. PloS one 2018, 13(2): 0191227.

. 32 Jamali AA, Ferdousi R, Razzaghi S, Li J, Safdari R, Ebrahimie E: DrugMiner: comparative analysis of machine learning algorithms for prediction of potential druggable proteins. Drug discovery today 2016, 21(5):718-724.

.33 Bakhtiarizadeh MR, Moradi-Shahrbabak M, Ebrahimi M, Ebrahimie E: Neural network and SVM classifiers accurately predict lipid binding proteins, irrespective of sequence homology. Journal of Theoretical Biology 2014, 356:213-222.

.34 Dillies M-A, Rau A, Aubert J, Hennequet-Antier C, Jeanmougin M, Servant N, Keime C, Marot G, Castel D, Estelle J: A comprehensive evaluation of normalization methods for Illumina high-throughput RNA sequencing data analysis. Briefings in bioinformatics 2013, 14(6):671-683. 
. 35 Caroli A, Chessa S, Erhardt G: Invited review: Milk protein polymorphisms in cattle: Effect on animal breeding and human nutrition. Journal of dairy science 2009, 92(11):5335-5352.

. 36 Fox P, Kelly A: Chemistry and biochemistry of milk constituents. Food Biochemistry and Food Processing 2006:425-452.

. 37 Bong JJ, Jeong JY, Rajasekar P, Cho YM, Kwon EG, Kim HC, Paek BH, Baik M: Differential expression of genes associated with lipid metabolism in longissimus dorsi of Korean bulls and steers. Meat science 2012, 91(3):284-293.

. 38 Ntambi JM, Miyazaki M: Recent insights into stearoyl-CoA desaturase-1. Current opinion in lipidology 2003, 14(3):255-261.

. 39 Bakhtiarizadeh MR, Moradi-Shahrbabak M, Ebrahimie E: Underlying functional genomics of fat deposition in adipose tissue. Gene 2013, 521(1):122-128.

. 40 Bionaz M, Loor JJ: Gene networks driving bovine milk fat synthesis during the lactation cycle. BMC genomics 2008, 9(1):366.

.41 Rodriguez-Cruz M, Tovar AR, Palacios-González B, del Prado M, Torres N: Synthesis of long-chain polyunsaturated fatty acids in lactating mammary gland: role of $\Delta \mathbf{5}$ and $\Delta \mathbf{6}$ desaturases, SREBP-1, PPARa, and PGC-1. Journal of lipid research 2006, 47(3):553-560.

. 42 Anderson SM, Rudolph MC, McManaman JL, Neville MC: Key stages in mammary gland development. Secretory activation in the mammary gland: it's not just about milk protein synthesis! Breast Cancer Research 2007, 9(1):204.

. 43 Varvikko T, Vanhatalo A, Jalava T, Huhtanen P: Lactation and metabolic responses to graded abomasal doses of methionine and lysine in cows fed grass silage diets. Journal of dairy science 1999, 
. 44 Coleman-Krnacik S, Rosen JM: Differential temporal and spatial gene expression of fibroblast growth factor family members during mouse mammary gland development. Molecular Endocrinology 1994, 8(2):218-229.

. 45 Provost E, Weier CA, Leach SD: Multiple ribosomal proteins are expressed at high levels in developing zebrafish endoderm and are required for normal exocrine pancreas development. Zebrafish 2013, 10(2):161-169.

. 46 Farhadian M, Rafat SA, Hasanpur K, Ebrahimi M, Ebrahimie E: Cross-species meta-analysis of transcriptomic data in combination with supervised machine learning models identifies the common gene signature of lactation process. Frontiers in genetics 2018, 9.

. 47 Tyagi S, Gupta P, Saini AS, Kaushal C, Sharma S: The peroxisome proliferator-activated receptor: A family of nuclear receptors role in various diseases. Journal of advanced pharmaceutical technology \& research 2011, 2(4):236.

. 48 Lin J, Bao ZK, Zhang Q, Hu WW, Yu QH, Yang Q: Transcriptome analysis of the mammary gland from GH transgenic goats during involution. Gene 2015, 565(2):228-234.

.49 Zhang C, Wang G, Wang J, Ji Z, Liu Z, Pi X, Chen C: Characterization and comparative analyses of muscle transcriptomes in Dorper and small-tailed Han sheep using RNA-Seq technique. PloS one 2013, 8(8):e72686.

.50 Caldeo V: Oxidation-reduction potential and its influence on Cheddar cheese quality. 2015.

. 51 Nath S, Villadsen J: Oxidative phosphorylation revisited. Biotechnology and bioengineering 2015, 112(3):429-437. 
. 52 McManaman JL, Neville MC: Mammary physiology and milk secretion. Advanced drug delivery reviews 2003, 55(5):629-641.

.53 Ebrahimie E, Ebrahimi F, Ebrahimi M, Tomlinson S, Petrovski KR: Hierarchical pattern recognition in milking parameters predicts mastitis prevalence. Computers and electronics in agriculture 2018, 147:611.

. 54 Pastushenko V, Matthes $\mathrm{H}$, Heinrich $\mathrm{H}$ : Effect of fat content on the redox potential behaviour of milk. Milchwissenschaft 2000, 55(10):547-549.

. 55 Berg JM, Tymoczko J, Stryer L: Glycolysis is an energy-conversion pathway in many organisms. Biochemistry 5th ed New York: WH Freeman 2002.

.56 Huston GE, Patton S: Factors related to the formation of cytoplasmic crescents on milk fat globules. Journal of dairy science 1990, 73(8):2061-2066.

. 57 Pacheco HA, da Silva S, Sigdel A, Mak CK, Galvão KN, Texeira RA, Dias LT, Peñagaricano F: Gene Mapping and Gene-Set Analysis for Milk Fever Incidence in Holstein Dairy Cattle. Frontiers in Genetics $2018,9(465)$.

. 58 Farhadian M, Rafat SA, Hasanpur K, Ebrahimi M, Ebrahimie E: Cross-Species Meta-Analysis of Transcriptomic Data in Combination With Supervised Machine Learning Models Identifies the Common Gene Signature of Lactation Process. Frontiers in Genetics 2018, 9(235).

.59 Farrell Jr H, Jimenez-Flores R, Bleck G, Brown E, Butler J, Creamer L, Hicks C, Hollar C, Ng-KwaiHang K, Swaisgood H: Nomenclature of the proteins of cows' milk-Sixth revision. Journal of dairy science 2004, 87(6):1641-1674. 

Nascimento J, Silva M: Sheep milk: Physicochemical characteristics and relevance for functional food development. Comprehensive reviews in food science and food safety 2017, 16(2):247-262.

. 61 Schanbacher F, Talhouk R, Murray F: Biology and origin of bioactive peptides in milk. Livestock Production Science 1997, 50(1-2):105-123.

.62 Silva SV, Malcata FX: Studies pertaining to coagulant and proteolytic activities of plant proteases from Cynara cardunculus. Food Chemistry 2005, 89(1):19-26.

.63 Apostoli AJ, Skelhorne-Gross GE, Rubino RE, Peterson NT, Di Lena MA, Schneider MM, SenGupta SK, Nicol CJ: Loss of PPARy expression in mammary secretory epithelial cells creates a pro-breast tumorigenic environment. International journal of cancer 2014, 134(5):1055-1066.

. 64 Kilgore KS, Billin AN: PPARbeta/delta ligands as modulators of the inflammatory response. Current opinion in investigational drugs (London, England: 2000) 2008, 9(5):463-469.

. 65 Spiegelman BM: PPAR-gamma: adipogenic regulator and thiazolidinedione receptor. Diabetes 1998, 47(4):507-514.

. 66 Crisà A, Ferrè F, Chillemi G, Moioli B: RNA-Sequencing for profiling goat milk transcriptome in colostrum and mature milk. BMC veterinary research 2016, 12(1):264.

\section{Figure Legends}

Fig 1

Expressed genes in MFGs at 20 and 70 days of lactation in Ghezel sheep. Log10 FPKM values are represented in the $\mathrm{X}$-axis and the gene names are represented in $\mathrm{Y}$-axis. A color code is used to present the two time points studied, red color for AF (after peak) and green color for BP (before peak) of lactation period. 
Fig 2

Volcano plot of the expressed genes in the two before and after peak lactation groups. The red points indicate the differentially expressed genes.

Fig 3

Gene Ontology enrichment of DEGs in two lactation stage. BP: Biological process, CC: Cellular component, MF: molecular function.

Fig 4

Expression changes in genes encoding whey protein, caseins and enzymes in lactose in BF and $\mathrm{AF}$ period of lactation in MFG samples of Ghezel sheep breed. Gene symbols are shown in X-axis and the log RPKMexpression values shown in Y-axis.

Fig 5

Expression changes in milk fat metabolism pathway genes during lactation. Stages of lactation and the FPKM value gene expression are shown in x-axis and $y$-axis respectively. The six part of fat metabolism in milk include: A) Fatty acid uptake, B) De novo fatty acid synthesis, C) Fatty acid desaturation, D) Fatty acid esterification, E) Regulation of fatty acid synthesis, F) Milk fat secretion. All of the genes in milk fat metabolism showed higher expression in BF than AF period. Gene names are in the legend.

Fig 6

STRING analysis shows that DEGs are involved in known and predicted protein-protein interactions. Network nodes represent genes. Lines of different color represent seven types of evidence used in predicting associations. Red line: fusion evidence; green line: neighborhood evidence; blue line: cooccurrence evidence; purple line: experimental evidence; yellow line: text mining evidence; light blue line: database evidence; black line: co-expression evidence. Interaction score > 0.4.

\section{Tables}

Due to technical limitations, tables are only available as a download in the supplemental files section. 


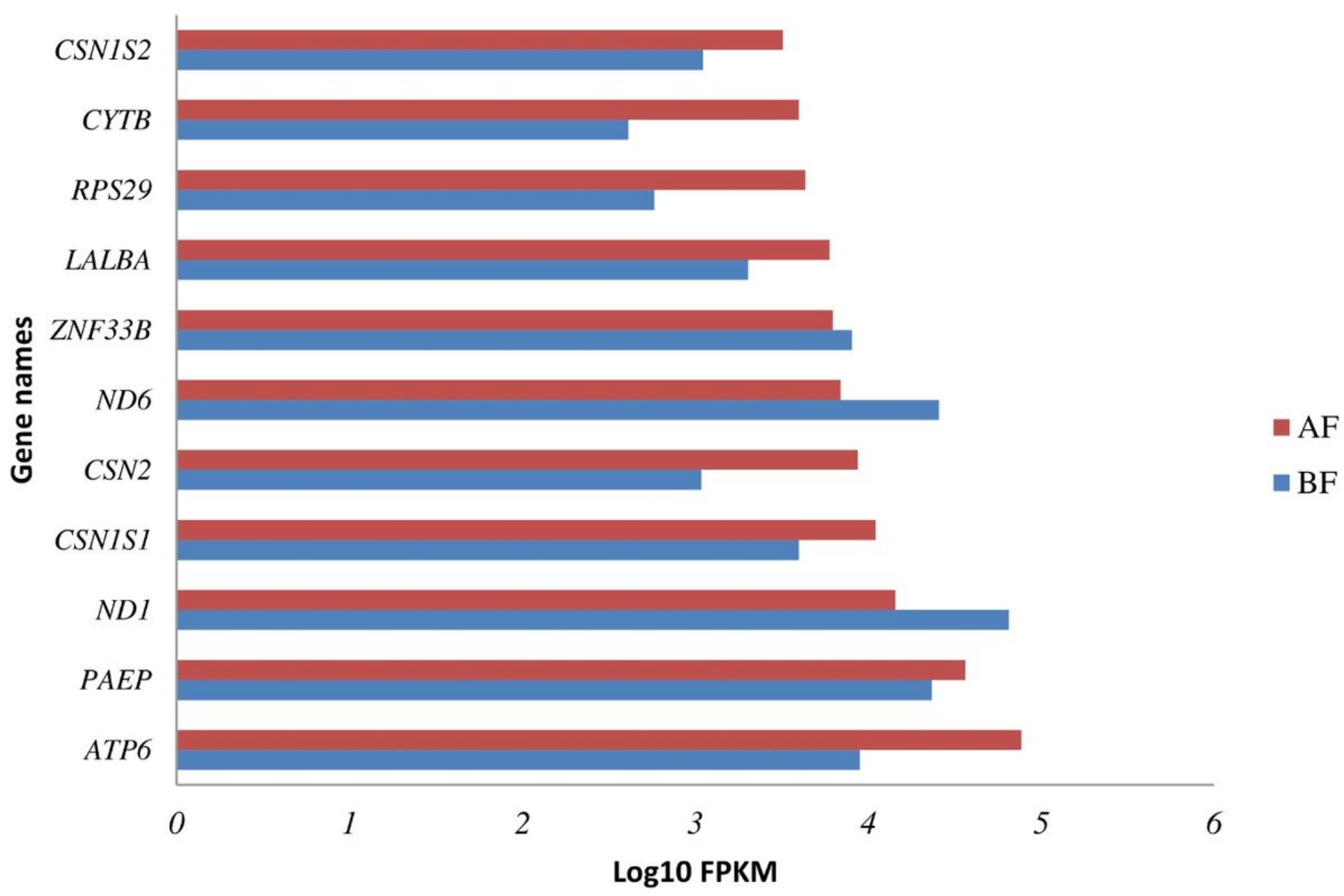

\section{Figure 1}

Expressed genes in MFGs at 20 and 70 days of lactation in Ghezel sheep. Log10 FPKM values are represented in the $\mathrm{X}$-axis and the gene names are represented in $\mathrm{Y}$-axis. A color code is used to present the two time points studied, red color for AF (after peak) and green color for BP (before peak) of lactation period. 


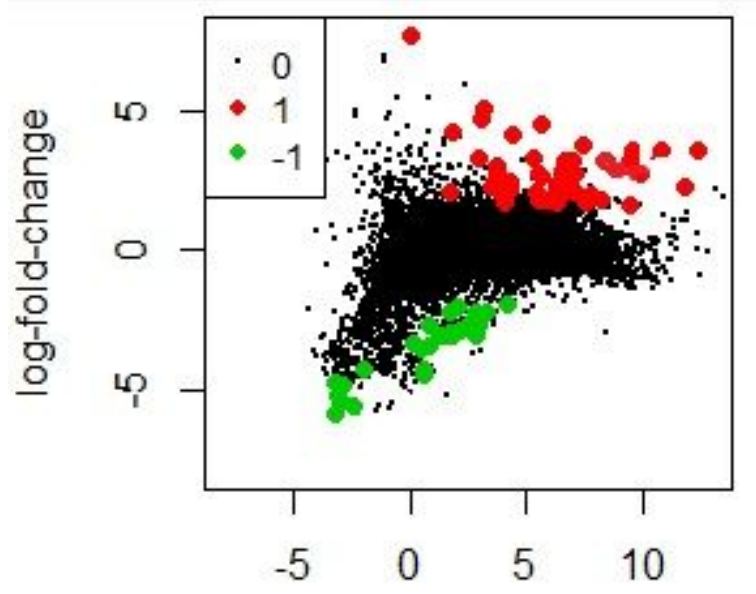

Average log-expression

\section{Figure 2}

Volcano plot of the expressed genes in the two before and after peak lactation groups. The red points indicate the differentially expressed genes. 

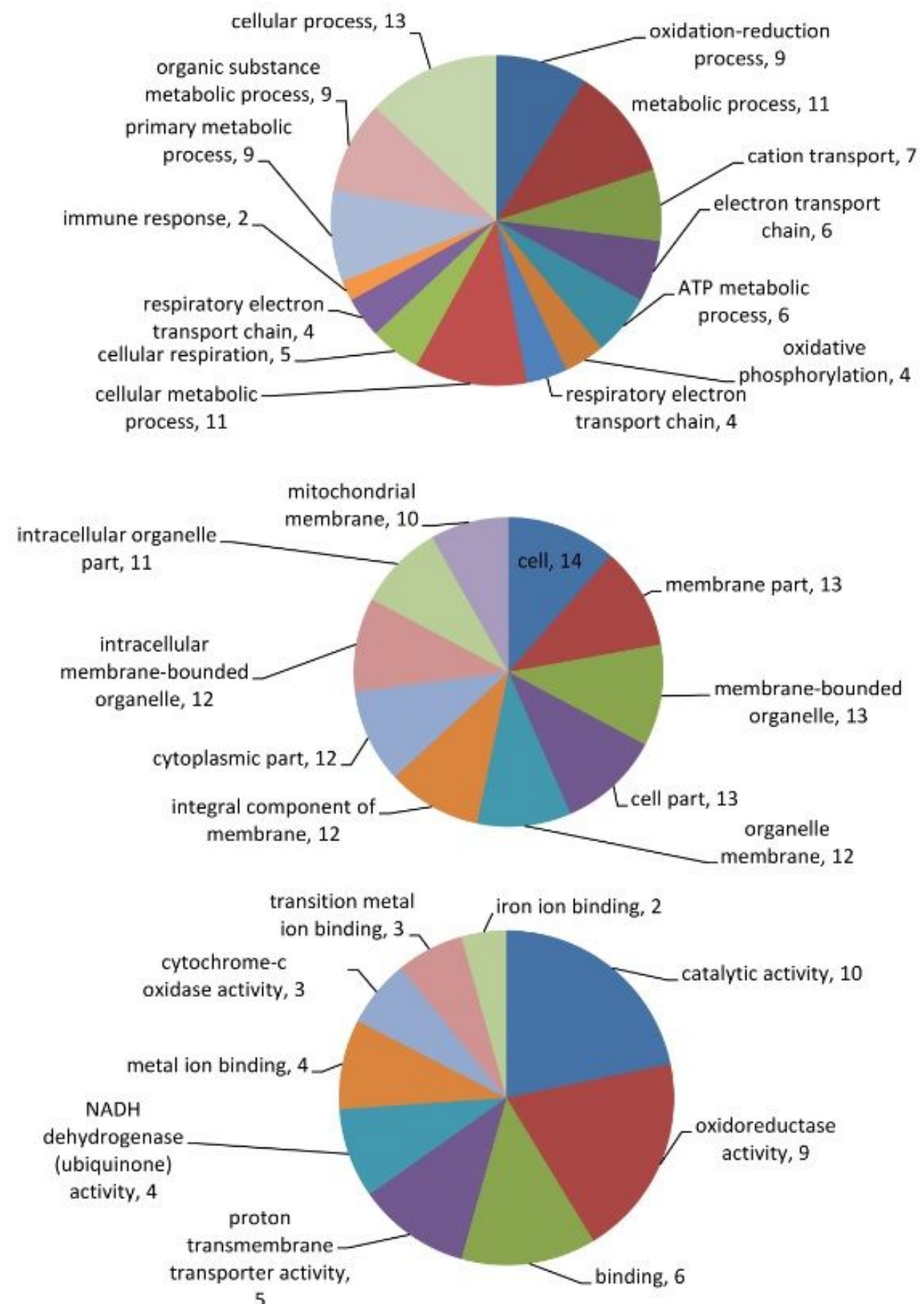

\section{Figure 3}

Gene Ontology enrichment of DEGs in two lactation stage. BP: Biological process, CC: Cellular component, MF: molecular function. 


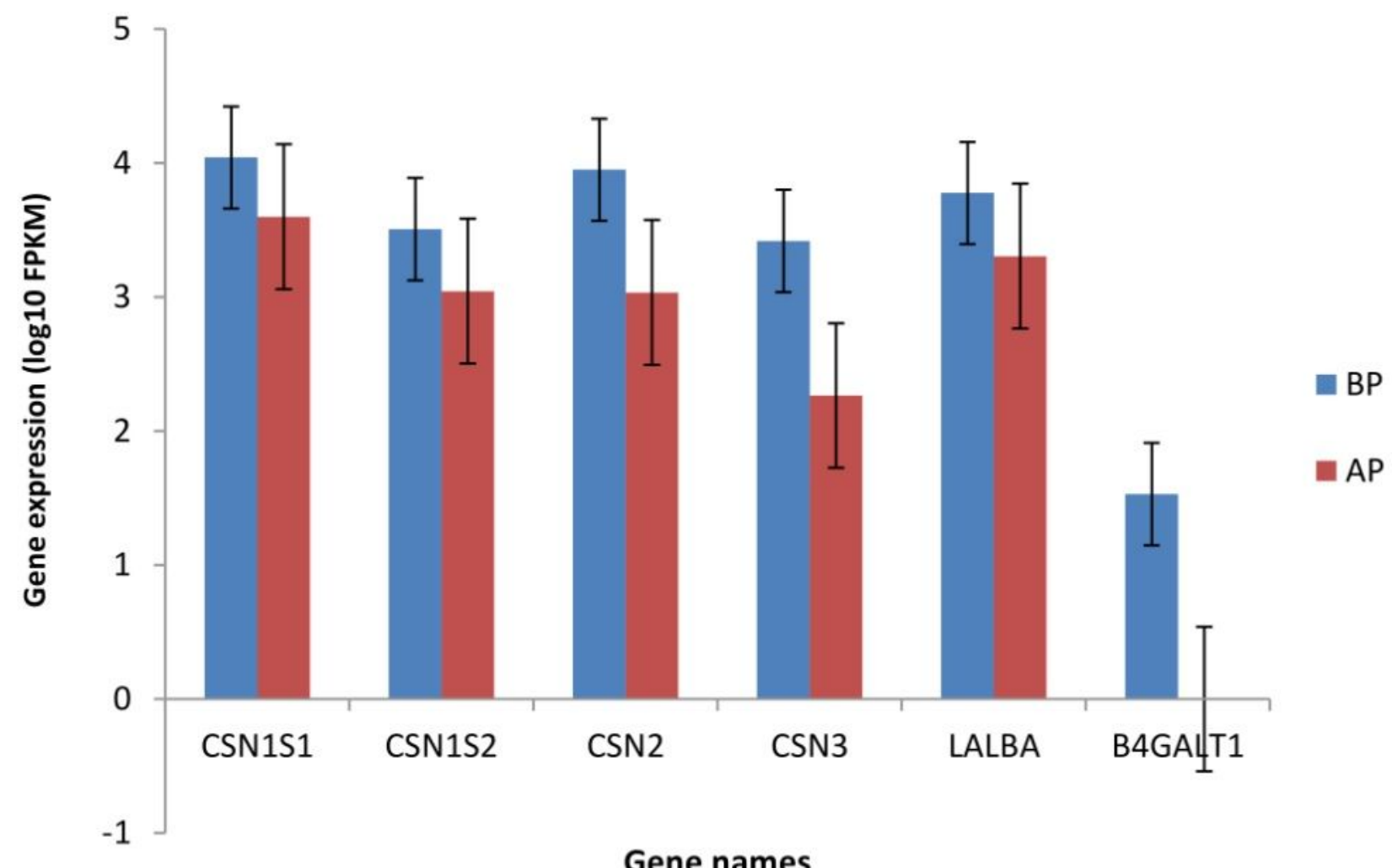

Figure 4

Expression changes in genes encoding whey protein, caseins and enzymes in lactose in BF and AF period of lactation in MFG samples of Ghezel sheep breed. Gene symbols are shown in X-axis and the log RPKMexpression values shown in Y-axis. 

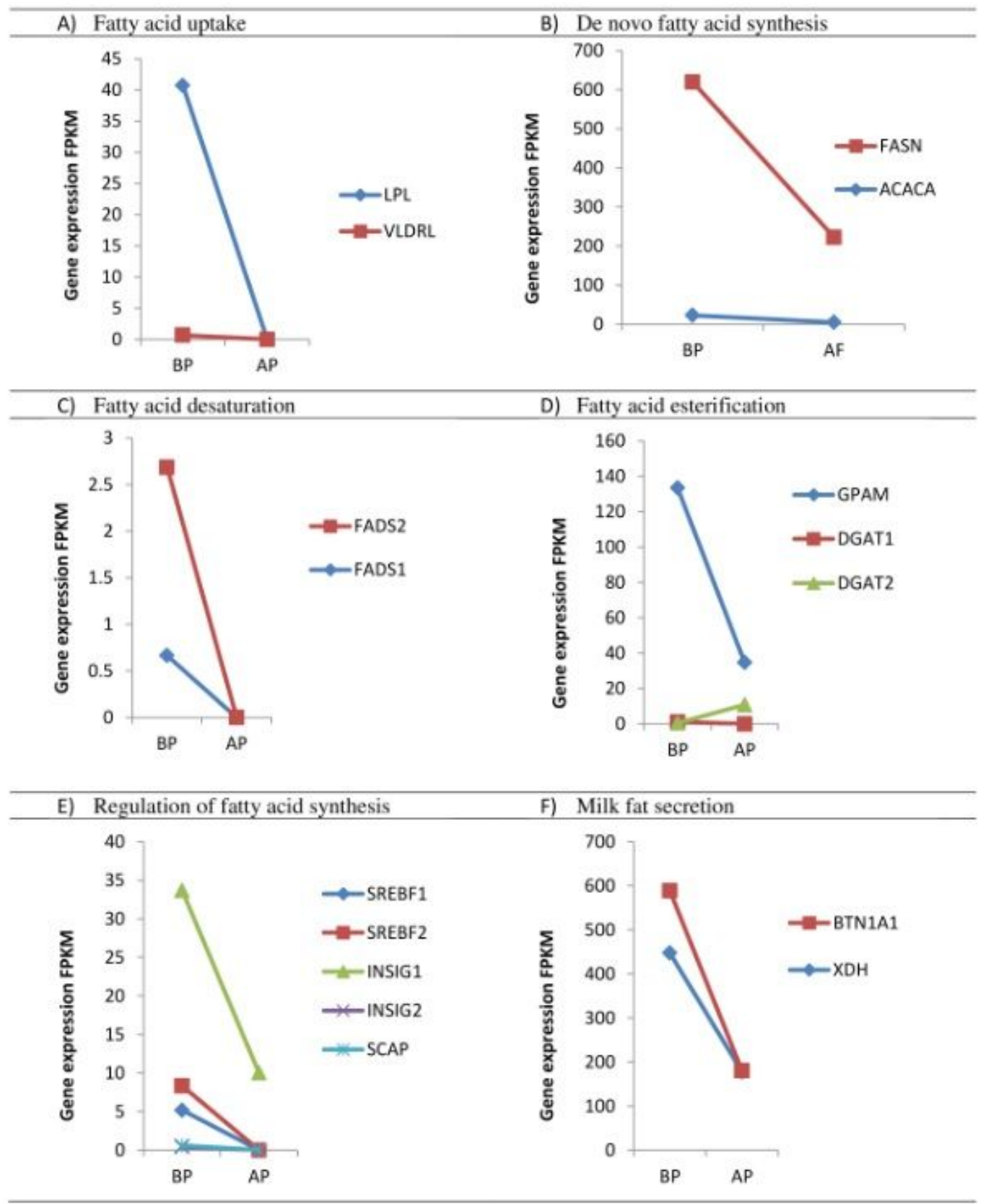

\section{Figure 5}

Expression changes in milk fat metabolism pathway genes during lactation. Stages of lactation and the FPKM value gene expression are shown in $x$-axis and $y$-axis respectively. The six part of fat metabolism in milk include: A) Fatty acid uptake, B) De novo fatty acid synthesis, C) Fatty acid desaturation, D) Fatty acid esterification, E) Regulation of fatty acid synthesis, F) Milk fat secretion. All of the genes in milk fat metabolism showed higher expression in BF than AF period. Gene names are in the legend. 


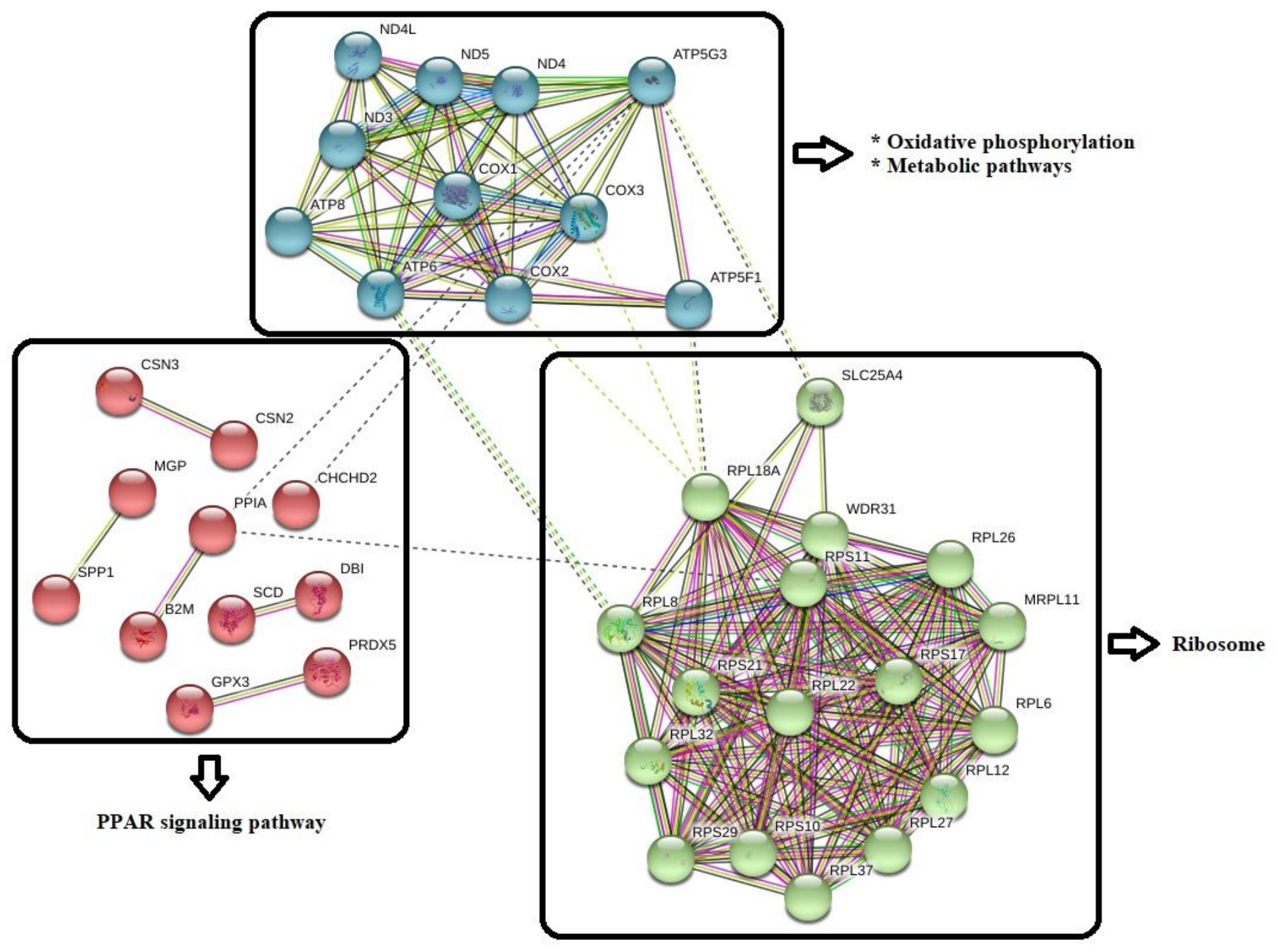

Figure 6

STRING analysis shows that DEGs are involved in known and predicted protein-protein interactions. Network nodes represent genes. Lines of different color represent seven types of evidence used in predicting associations. Red line: fusion evidence; green line: neighborhood evidence; blue line: cooccurrence evidence; purple line: experimental evidence; yellow line: text mining evidence; light blue line: database evidence; black line: co-expression evidence. Interaction score > 0.4 .

\section{Supplementary Files}

This is a list of supplementary files associated with this preprint. Click to download.

- supplement1.pdf

- supplement2.pdf

- supplement3.pdf 